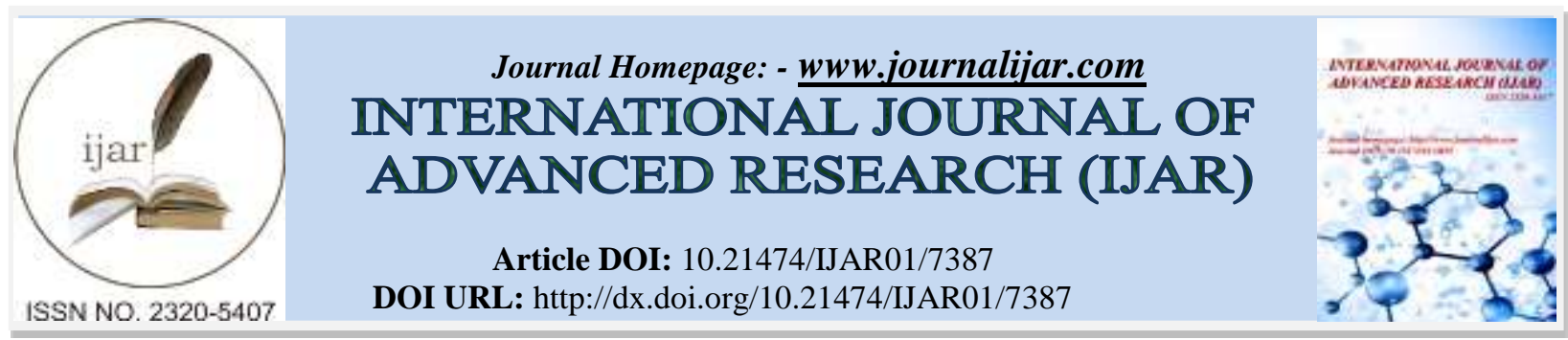

RESEARCH ARTICLE

\title{
A NEW SPECIES OF DIMERIA (POACEAE: PANICOIDEAE), FROM MIDDLE ANDAMAN, INDIA.
}

\section{Pushpa Kumari and Reshma Lakra.}

Central National Herbarium, Botanical Survey of India, P.O. Botanic Garden, Howrah-711103, West Bengal, India.

\section{Manuscript Info}

Manuscript History

Received: 10 May 2018

Final Accepted: 12 June 2018

Published: July 2018

Keywords:-

Andaman Islands, branched, Dimeria fasciculata, New species.
Abstract

Dimeria fasciculata (Poaceace) collected from Panchvati, Middle Andaman of the Andaman group of Islands in India is described and illustrated as a new species. Some of its morphological characters resemble with other members of the genus Dimeria avenacea, Dimeria fuscescens, Dimeria kurzii and Dimeria ornithopoda but this species differs from all in having culms branched on almost all nodes; branches $3-9$, unequal in length, terminating usually in solitary, rarely 2-digitate racemes; spikelets embedded within the rachis; and awn of lemma short, stiff, aligned along with raceme. Morphological features place this species under Section Dimeria of Genus Dimeria.

Copy Right, IJAR, 2018. All rights reserved.

\section{Introduction:-}

Dimeria R. Br., a paleotropical genus belongs to monotypic subtribe Dimeriinae Hack. (Poaceae, Panicoideae, Andropogoneae). Although there is a difference of opinion regarding number of species, 59 species under this genus (Mabberley, 2017) are reported from the world. The species are found all over the tropical Asian regions from India to China, Korea, Japan, Indonesia, the Philippines, Northern Australia, and to Sri Lanka and Madagascar (Bor 1953; Teerawatananon et. al. 2014; Kiran Raj et. al. 2015a; Naik M.C. et. al. 2016) of which c. 40 species are present in India (Ravi et. al. 2001; Kiran Raj et. al. 2015a, Gosavi et. al. 2016).

The genus is characterized by its usually equal and divergent binate racemes with laterally compressed, homogamous, 1-fl diandrous spikelets. Bor (1952), based on the rachis characters classified the genus Dimeria R. Br. into three sections: Capillares, loriformes and Annulares. Later Kiran Raj et al. (2015) proposed a new section Dimeria with eleven species. Sect. Dimeria is characterized by triquetrous, trigonous, or angular, wingless or minutely winged rachis being scaberulous to sparsely ciliate along margins; usually reddish-purple or fuscous, imbricate spikelets and sub-coriaceous glumes. 12 species from sect. Dimeria have been reported from India (Kiran Raj 2008; Kiran Raj et. al. 2015b).

Corresponding Author: - Pushpa Kumari

Address: - Central National Herbarium, Botanical Survey of India, P.O.: Botanic Garden, Howrah - 711103, West Bengal, INDIA. 
From Andaman and Nicobar Islands three species namely Dimeria andamanica Gosavi, M.Y. Kamble, Chandore \& S.R.Yadav under sect. Loriformes Bor, Dimeria hohenackeri Hochst. ex Miq. under sect. Capillares Bor ex Kiran Raj et Sivad., and Dimeria ornithopoda Trin. under sect. Dimeria are reported (Pandey \& Diwakar 2008; Gosavi et al. 2016; Naik et al. 2016).

During the exploration tour to Andaman and Nicobar Islands in 2017, an interesting species of Dimeria was collected from Panchvati, Middle Andaman. Intensive study of the herbarium specimens and thorough study of related literature (Bor 1960; Clayton \& Renvoize 1986; Ravi et. al. 1995; Mohanan \& Ravi 1996; Ravi et. al. 1997; Ravi et. al. 2001; Kiran Raj \& Sivdasan 2008; Arisdason \& Daniel 2009; Kabeer \& Nair 2010; Teerawatananon et. al. 2014; Biju et. al. 2017), revealed that collected Dimeria species is unique in its morphological characters in having culms branched on almost all nodes in fascicle of 3-9 and terminating into single, rarely 2-digitate racemes. Thus, the current collection is described as a distinct new species.

Taxonomy: - Dimeria fasciculata P. Kumari \& R. Lakra sp. nov. Fig. 1 \& 2.

Type: - India. Andaman Islands: Middle Andaman, Panchvati, Long. E92 58'11.9", Lat. N12³3'13.4", 08 February 2017, Reshma Lakra 32835 (holotype CAL!; isotype PBL!).

\section{Diagnosis: -}

Dimeria fasciculata resembles with Dimeria avenacea (Retz.) C.E.C.Fisch., Dimeria fuscescens Trin., Dimeria kurzii Hook.f., and Dimeria ornithopoda but differs from all in having culms branched on almost all nodes; branches 3-9, unequal, terminating usually in solitary, rarely 2-digitate racemes; spikelets embedded within the rachis; and awn of lemma short, stiff, aligned along with raceme.

\section{Description: -}

Tufted annuals. Culms 8-35 cm tall, geniculate, branched on almost all nodes; nodes swollen, bearded with ring of fine white hair; hair up to $0.8 \mathrm{~mm}$ long; internodes $1-3.6 \mathrm{~cm} \times 0.5-1 \mathrm{~mm}$, terete, glabrous; branches 3-9, unequal in length. Leaf blades $3-8 \mathrm{~cm} \times 1-1.8 \mathrm{~mm}$, linear, acute at apex, ventral surface faintly scabrid, margins with bulbous based 1-3 mm long hair; ligule membranous, obtuse or truncate, praemorse; leaf sheaths 1-2 cm long, compressed, mid-rib prominent, margin papery. Peduncle $0.5-9.5 \mathrm{~cm}$ long, glabrous terete. Raceme solitary or rarely 2-digitate, unilateral, 3-6 cm long. Rachis $0.3-0.4 \mathrm{~mm}$ wide, tough, triquetrous with alternately arranged spikelets, ridges scabrid. Spikelets oblong-elliptic, scabrid, hairy, awned, brownish, closely attached to rachis. Lower glume 2-2.5 $\mathrm{mm} \times 0.3-0.4 \mathrm{~mm}$, narrowly oblong to linear, compressed, coriaceous, acute at apex; keeled and narrowly winged, scabrellate; surface puberulous and scabrid; margins open. Upper glume $2.5-3 \mathrm{~mm} \times 0.4-0.5 \mathrm{~mm}$, oblong, compressed, coriaceous, acute to acuminate at apex; keeled and narrowly winged, scabrellate; surface puberulous and scabrid; margins open, ciliate on upper half. Florets-2, lower barren, upper bisexual. Lower lemma $0.8-1 \mathrm{~mm} \times$ 0.1-0.2 mm, lanceolate, membranous. Lower palea absent. Upper lemma 1.8-2.2 $\mathrm{mm} \times 0.3-0.4 \mathrm{~mm}$, lanceolate to oblong, membranous, apex with $0.1 \mathrm{~mm}$ incision, awned; awn arising from the sinus, $0.3-3.5 \mathrm{~mm}$ long, scabrid. Upper palea absent. Lodicules-2, 0.2-0.3 mm long, hyaline. Stamens-2, 0.5-0.8 mm $\times 0.2-0.3 \mathrm{~mm}$. Ovary 0.5-1 $\mathrm{mm} \times 0.2 \mathrm{~mm}$, oblong. Stigmas-2, up to $1 \mathrm{~mm}$ long, plumose. Caryopsis $1.5-2 \mathrm{~mm} \times 0.2-0.3 \mathrm{~mm}$, oblong, brownish yellow, with persistent style. Fig. 1, 2 .

\section{Distribution: -}

India, Andaman Islands.

\section{Specimens Examined: -}

INDIA. Andaman Islands: Middle Andaman, Panchvati, 08 Feb. 2017, Long. E92 58'11.9", Lat. N12³3'13.4", Reshma Lakra 32835 (holotype CAL; isotype PBL). 


\section{Habitat \& Ecology: -}

The species grows on rocky surface of hill slopes along fringes of semi-evergreen forests, with associates like Paspalum scrobiculatum L., Schizostachyum andamanicum M.Kumar \& Remesh, and Pennisetum sp.

\section{Conservation Status: -}

Data Deficient (IUCN 2001). The species is currently reported only from the type locality in a restricted fragmented rocky area with small population in dense patches. As it is a new species being described here, a proper status can only be assigned after further exploration and reports of the species.

\section{Flowering \& Fruiting: -}

November to February.

\section{Etymology:-}

The specific epithet is named after the fascicled nature of branches of this plant.

Notes: -

Habit appearance of D. fasciculata is unique due to its multiple branched culm and further branched branch nodes, which makes it congested in appearance and distinguish this species from all other members at once.

\section{Recognition: -}

Dimeria fasciculata is characterized by triquetrous, scaberulous rachis margins, sub-coriaceous glumes and belongs to genus Dimeria of sect. Dimeria. It resembles D. avenacea, D. fuscescens, D. kurzii and D. ornithopoda but differs in having culm branched on almost all nodes; branches unequal in length, in groups of 3-9; raceme solitary or rarely 2-digitate, unilateral; spikelets embedded within the rachis; awn of lemma minute, stiff, along with raceme. A detailed comparison is shown in Table 1. and taxonomic identification key of all related species is given below:

\section{Key to the species}

1a. Branches 3-9, fascicled; spikelets embedded within the rachis; awn of lemma $0.3-3.5 \mathrm{~mm}$ long, stiff, straight, aligned along with raceme.

D. fasciculata

1b. Branches 0-3, not fascicled; spikelets exerted from the rachis; awn of lemma 4-15 mm long, twisted, geniculate, spreading out of raceme. 2

2a. Culm unbranched; racemes $5-12 \mathrm{~cm}$ long. D. fuscescens

2b. Culm branched; racemes $1-5 \mathrm{~cm}$ long. 3

3a. Raceme usually 2 , rarely 1,3 , or 4 ; upper glume slightly keeled towards apex; upper lemma awn 4-5 mm long.

D. ornithopoda

3b. Raceme usually solitary, rarely 2; upper glume keeled from base to apex; upper lemma awn $8-18 \mathrm{~mm}$ long. 


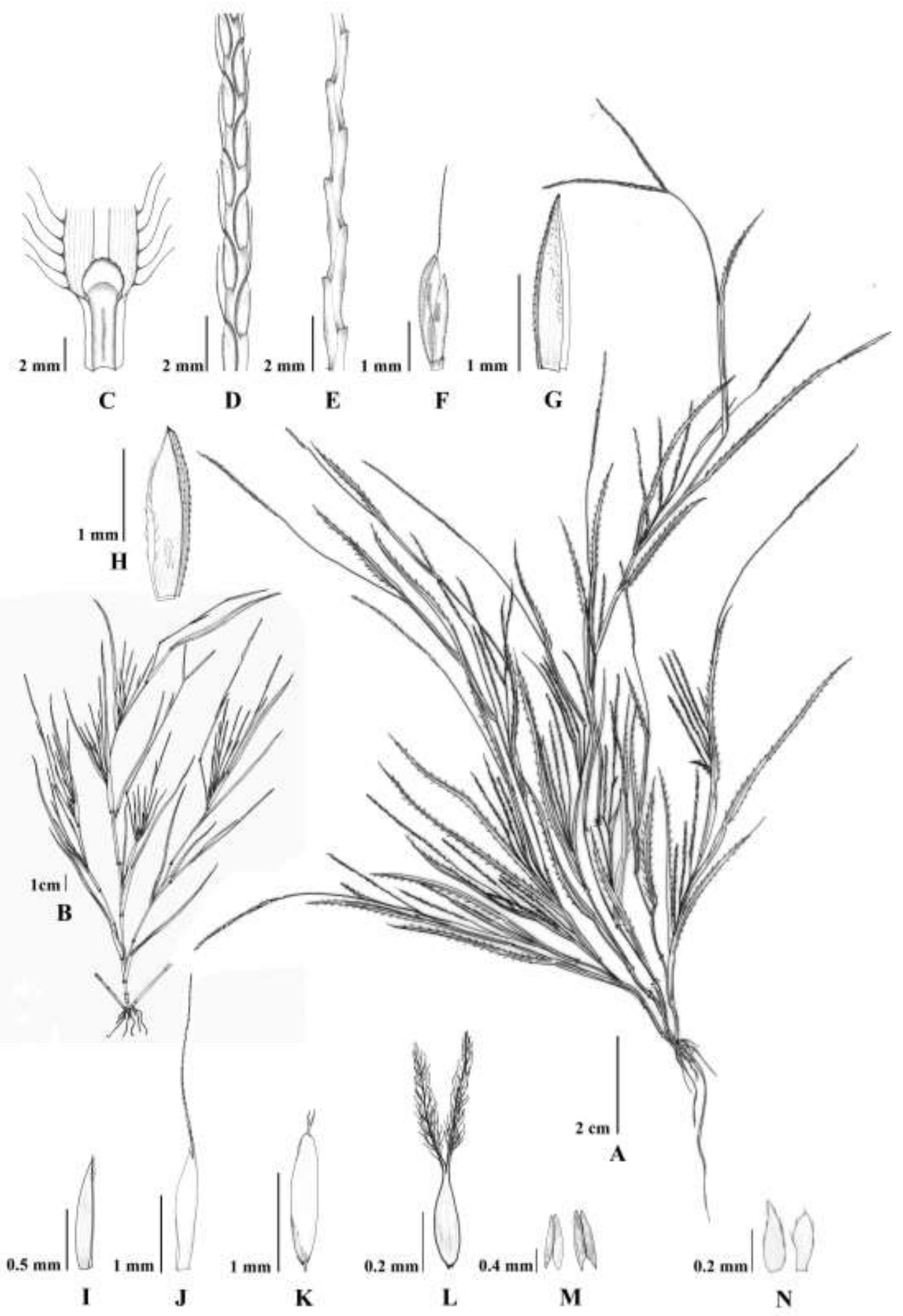

Figure 1: - Dimeria fasciculata. A Habit; B Branching pattern; C Ligule; D Raceme; E Rachis; F Spikelet; G Lower glume; H Upper glume; I Lower lemma; J Upper lemma; K Caryopsis; L Gynoecium; M Anther; N Lodicules 


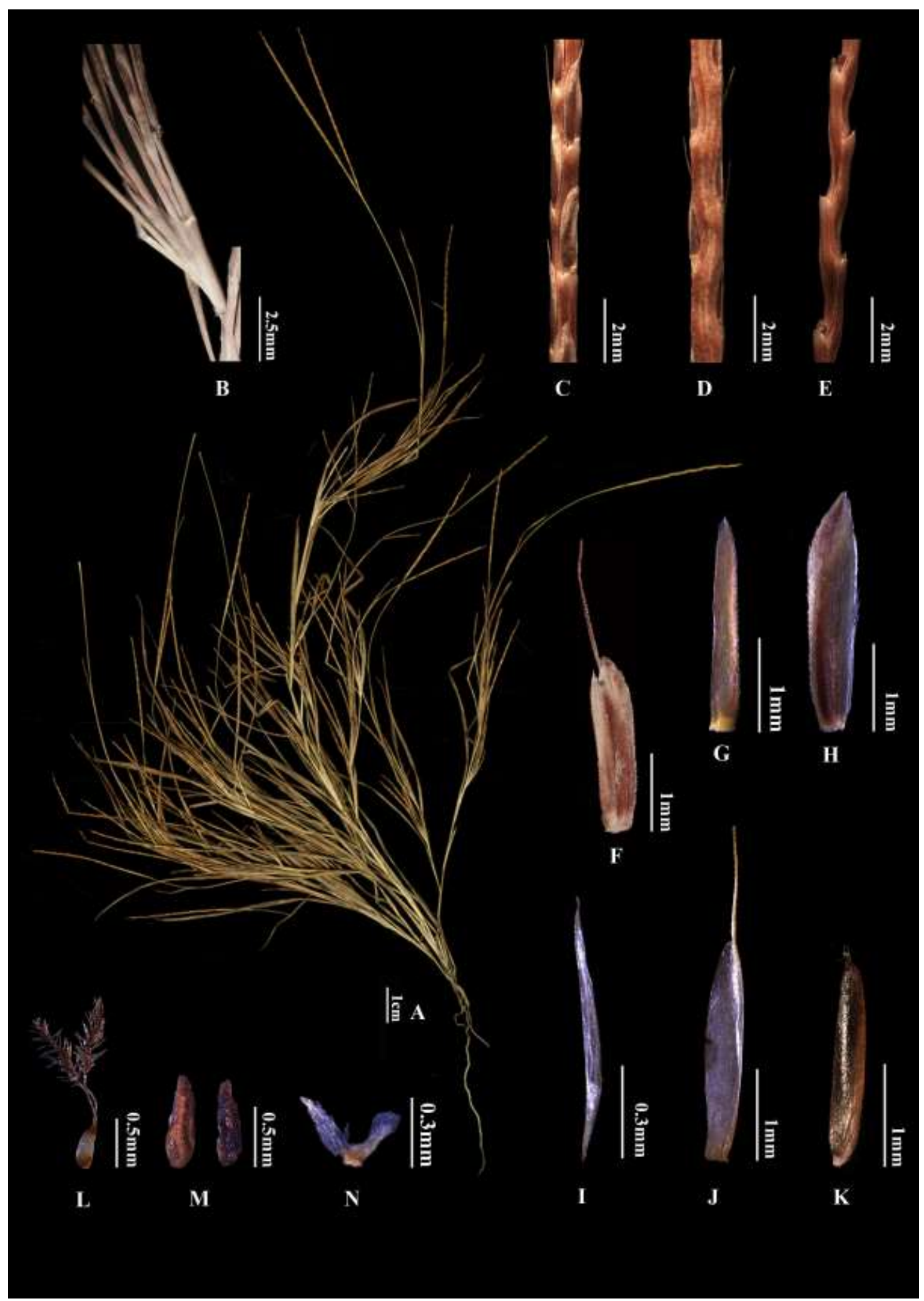

Figure 2: - Dimeria fasciculata. A Habit; B Branching pattern; C Part of raceme (Ventral); D Part of raceme (Dorsal); E Rachis; F Spikelet; G Lower glume; H Upper glume; I Lower lemma; J Upper lemma; K Caryopsis; L Gynoecium; M Anther; $\mathbf{N}$ Lodicules 
Table 1: - Distinguishing character of Dimeria fasciculata with D. kurzii, D. avenacea, D. fuscescens and D. ornithopoda.

\begin{tabular}{|c|c|c|c|c|c|}
\hline Characters & D. fasciculata & D.kurzii & D. avenacea & D. fuscescens & D. ornithopoda \\
\hline Culms & $\begin{array}{l}\text { Caespitose, } \\
\text { branched, } \\
\text { branches } \\
\text { fascicled 3-9 }\end{array}$ & $\begin{array}{l}\text { Caespitose, } \\
\text { branched, } \\
\text { branches } 2\end{array}$ & Caespitose, solitary & $\begin{array}{l}\text { Solitary, branched, } \\
\text { branches } 2\end{array}$ & $\begin{array}{l}\text { Caespitose, } \\
\text { branched, } \\
\text { branches 1-5 }\end{array}$ \\
\hline Raceme & $\begin{array}{l}\text { Solitary or } \\
\text { rarely 2-digitate, } \\
3-6 \mathrm{~cm} \text { long }\end{array}$ & $\begin{array}{l}\text { Solitary, } 2-5 \mathrm{~cm} \\
\text { long }\end{array}$ & $\begin{array}{l}\text { Solitary, } 4-4.5 \mathrm{~cm} \\
\text { long }\end{array}$ & $\begin{array}{l}2 \text {, rarely } 3 \text { or } 4- \\
\text { digitate, } 5-12 \mathrm{~cm} \\
\text { long }\end{array}$ & $\begin{array}{l}\text { 2, rarely } 1,3 \text { or } \\
\text { 4- digitate, } 1-5 \\
\mathrm{~cm} \text { long }\end{array}$ \\
\hline Rachis & $\begin{array}{l}0.3-0.4 \quad \mathrm{~mm} \\
\text { wide }\end{array}$ & $0.4-0.8 \mathrm{~mm}$ wide & $0.4-0.7 \mathrm{~mm}$ wide & $0.2-0.3 \mathrm{~mm}$ wide & $\begin{array}{l}0.25-0.5 \quad \mathrm{~mm} \\
\text { wide }\end{array}$ \\
\hline $\begin{array}{l}\text { lower } \\
\text { glume }\end{array}$ & $\begin{array}{l}2-2.5 \mathrm{~mm} \text { long, } \\
\text { keeled and } \\
\text { winged, acute } \\
\text { at apex }\end{array}$ & $\begin{array}{l}2-2.3 \mathrm{~mm} \text { long, } \\
\text { not keeled but } \\
\text { slightly } \\
\text { compressed at } \\
\text { acuminate or } \\
\text { shortly aristate } \\
\text { apex }\end{array}$ & $\begin{array}{l}4-5 \mathrm{~mm} \text { long, } \\
\text { keeled towards } \\
\text { short awned apex }\end{array}$ & $\begin{array}{l}4-4.5 \mathrm{~mm} \text { long, } \\
\text { keeled, acuminate } \\
\text { at apex }\end{array}$ & $\begin{array}{l}0.8-2 \mathrm{~mm} \text { long, } \\
\text { keeled, acute } \\
\text { at apex }\end{array}$ \\
\hline $\begin{array}{l}\text { Upper } \\
\text { glume }\end{array}$ & $\begin{array}{l}2.5-3 \mathrm{~mm} \text { long, } \\
\text { apex acute to } \\
\text { acuminate, } \\
\text { keeled and } \\
\text { winged from } \\
\text { base to apex }\end{array}$ & $\begin{array}{l}2.1-2.5 \quad \mathrm{~mm}, \\
\text { slightly } \\
\text { compressed at } \\
\text { bifid shortly } \\
\text { aristate apex }\end{array}$ & $\begin{array}{l}4.5-5 \mathrm{~mm} \text { long } \\
\text { (excl. awn), awned, } \\
\text { winged from base } \\
\text { to apex }\end{array}$ & $\begin{array}{l}4.4-5 \mathrm{~mm} \text { long, } \\
\text { apex } \\
\text { narrowly winged } \\
\begin{array}{l}\text { and } \\
\text { towards apex }\end{array}\end{array}$ & $\begin{array}{l}1-2.5 \mathrm{~mm} \text { long, } \\
\text { apex acute, } \\
\text { keeled at apex }\end{array}$ \\
\hline $\begin{array}{l}\text { Lower } \\
\text { lemma awn }\end{array}$ & $\begin{array}{l}0.3-3.5 \quad \mathrm{~mm} \\
\text { long }\end{array}$ & 12-18 mm long & 8-13 mm long & $10-15 \mathrm{~mm}$ long & 4- $5 \mathrm{~mm}$ long \\
\hline
\end{tabular}

\section{References: -}

1. Arisdason, W. \& Daniel, P. (2009). Dimeria jayachandranii (Poaceae), a new species from Western Ghats, India. Kew Bulletin 64: 345-347.

2. Biju, P., Josekutty E. J. \& Jomy Augustine (2017). A new remarkable species of Dimeria R. Br. (Poaceae, Panicoideae, Andropogoneae) from the lateritic plateaus of northern Kerala, India. Int. J. Adv. Res. 5(11): 705711.

3. Bor, N.L. (1953). Notes on Asiatic Grasses Dimeria R.Br. in India and Burma. Kew Bulletin 7 (4): 553-592.

4. Bor, N.L. (1960). The Grasses of Burma, Ceylon, India and Pakistan. Pergamon Press, London, 767 pp.

5. Clayton, W.D. \& Renvoize, S.A. (1986). Genera Graminum: Grasses of the World. Her Majesty's Stationary Office, London, $389 \mathrm{pp}$.

6. Gosavi, K.V.C., Kamble, M.Y., Chandore, A.N., \& Yadav, S.R. (2016). A new species of Dimeria (Poaceae) from Andaman and Nicobar Islands, India. Phytotaxa 270 (4): 295-300.

7. IUCN. (2001). IUCN Red List Categories: Version 3.1. Published on the Internet; http//www.iucn.redlist.org

8. Kabeer, A. A. \& Nair, V. J. (2010). Flora of Tamil Nadu Grasses pp. 148-149. Botanical Survey of India, Coimbatore. 
9. Kiran Raj, M.S. (2008). Taxonomic Revision of the subtribe Dimeriinae Hack. of Andropogoneae (PoaceaePanicoideae) in Peninsular India. Ph.D. thesis (unpublished). University of Calicut, India.

10. Kiran Raj, M.S. \& Sivadasan, M. (2008). A new species of Dimeria R.Br (Poaceae: Panicoideae Andropogoneae) from Goa, India. Novon 18: 183-186.

11. Kiran Raj, M.S., Sivadasan, M., Alfarhan, A.H., \& Veldkamp, J.F. (2015a). Dimeria raviana (Poaceae: Panicoideae), a new species from Southern Western Ghats, India. Phytotaxa 195 (2): 193-196.

12. Kiran Raj, M.S., Sivadasan, M., Veldkamp, J.F., Alfarhan, A.H. \& Amal Tamimi, A.S.M. (2015b). A Revised Infrageneric Classification of Dimeria R.Br. (Poaceae: Andropogoneae). Bangladesh Journal of Plant Taxonomy. 22(1): 47-54.

13. Mabberley, D.L. (2017) Mabberley's Plant-book, $4^{\text {th }} e d$. Cambridge University Press. Cambridge, UK, XVIII + $1102 \mathrm{pp}$.

14. Mohanan, N. \& Ravi, N (1996). Dimeria Sivaranjini (Poaceae) from Kerala, India, Blumea 41: 251-256.

15. Naik, M.C., M.A.Kumar \& B.R.P.Rao (2016). On the discovery of Dimeria hohenackeri (Poaceaee) from Andaman Islands, a hitherto known endemic and endangered grass species of southwestern peninsular India. Journal of Threatened Taxa 8 (14): 9678-9680.

16. Pandey, R.P. \& Diwakar, P.G. (2008). An Integrated Check-list flora of Andaman and Nicobar Islands, India. Journal of Economy and Taxonomy Botany 32 (2): 403-500.

17. Ravi, N. (1996). Another two new species of Dimeria (Poaceae) from Kerala, India. Blumea 41: 251-256.

18. Ravi, N. \& Mohanan, N. (1997). Dimeria namboodriana, another new species of Poaceae from Kerala, India. Rheedea 7 (1): 1-4.

19. Ravi, N. \& Mohanan, N. \& Kiran Raj, M.S. (2001). Three new species of Poaceae from South India. Rheedea 11: 87-96.

20. Ravi, N., Saxena, H.O. \& Brahmam, N. (1995) Dimeria mahendragiriensis-A new species of Poaceae from Orissa, India. Rheedea 5 (2): 142-144.

21. Teerawatananon, A., Boontia, Chantarasuwan, B., Hodkinson, T.R. \& Sungkaew, S. (2014) A taxonomic revision of the genus Dimeria (Poaceae: Panicoideae) in Thailand. Phytotaxa 186 (3): 137-147. 